1 Title: A single amino acid substitution (H451Y) in Leishmania calcium-dependent kinase

2 SCAMK confers high tolerance and resistance to antimony.

3 Running title: Leishmania tolerance and resistance

5 Authors: Baptiste VERGNES ${ }^{1 *}$, Elodie GAZANION ${ }^{1}$, Cédric MARIAC ${ }^{2}$, Miléna DU MANOIR ${ }^{1}$, Lauriane SOLLELIS ${ }^{1}$, José-Juan LOPEZ-RUBIO ${ }^{1}$, Yvon STERKERS ${ }^{1,3}$, Anne-Laure BAÑULS ${ }^{1}$

8 Contact Information: ${ }^{1}$ MIVEGEC, IRD, CNRS, Univ. Montpellier, Montpellier, France; ${ }^{2}$ DIADE, 9 IRD, Univ. Montpellier, Montpellier, France; ${ }^{3}$ Department of Parasitology-Mycology, Faculty 10 of Medicine, University Hospital Center of Montpellier, Univ. Montpellier, Montpellier, France.

Present address: Lauriane Sollelis, Wellcome Centre for Molecular Parasitology, Institute of Infection, Immunity \& Inflammation, College of Medical, Veterinary and Life Sciences,

14 University of Glasgow, Glasgow, UK

*Corresponding author: Baptiste Vergnes Ph.D., UMR MIVEGEC (IRD, CNRS, Univ. Montpellier), 911 Avenue Agropolis, 34394 Montpellier Cedex 5, France. Phone number: +33(0)467416308. Email address: baptiste.vergnes@ird.fr

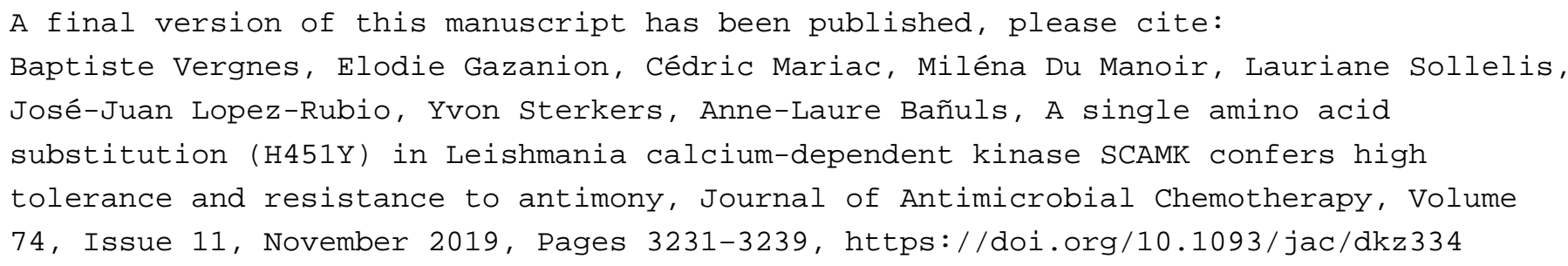


Synopsis:

Background: For almost a century, antimonials remain the first-line drugs for the treatment of leishmaniasis. However, little is known about their mode of action and clinical resistance mechanisms.

Objectives: We have previously shown that Leishmania nicotinamidase (PNC1) is an essential enzyme for parasite NAD+ homeostasis and virulence in vivo. Here, we found that parasites lacking $p n c 1$ gene ( $\Delta p n c 1)$ are hypersusceptible to the active form of antimony (SbIII) and used these mutant parasites to better understand antimony mode of action and resistance.

Methods: Sblll-resistant WT and $\Delta p n c 1$ parasites were selected in vitro by stepwise selection method. NAD $(H) / N A D P(H)$ dosages and quantitative RT-PCR experiments were performed to explain the susceptibility differences observed between strains. WGS and a marker-free CRISPR/Cas9 base editing approach were used to identify and validate the role of a new resistance mutation.

Results: NAD+ depleted $\Delta p n c 1$ parasites are highly susceptible to SbIII and this phenotype can be rescued by NAD+ precursor of trypanothione precursors supplementation. $\Delta p n c 1$ parasites can become resistant to SbIII by unknown mechanism. WGS revealed a unique amino acid substitution (H451Y) in an EF-hand domain of an orphan calcium-dependent kinase recently named SCAMK. When introduced into a wild type reference strain by base editing, the H451Y mutation allows Leishmania parasites to survive at extreme concentrations of SbIII, potentiating the rapid emergence of resistant parasites.

Conclusions: These results establish that Leishmania SCAMK is a new central hub of antimony mode of action and resistance development and uncover the importance of drug tolerance mutations in the evolution of parasite drug resistance. 
Leishmania are protozoan parasites transmitted by sandflies that are responsible for a wide spectrum of human infections ranging from the life-threatening visceral disease to disfiguring mucosal and cutaneous forms ${ }^{1}$. Treatment of leishmaniasis is limited to four main drugs (antimonials, miltefosine, amphotericin B and paromomycin). Alone or in combination, antimonials have been the mainstay of anti-Leishmania therapy worldwide for over 70 years. However, our knowledge of antimony mode of action is still partial and the emergence of parasite resistance to antimony in the Indian subcontinent during the last decades has necessitated the use of alternative medications. The active form of antimony (SbIII) is known to target the Leishmania redox potential by inducing rapid thiols efflux and inhibiting the NADPH-dependent trypanothione reductase (TR) that maintains the main parasite thiol, trypanothione, in its reduced form ${ }^{2}$ (Figure 1a). Leishmania resistance to Sblll is generally mediated by drug uptake reduction through the aquaglyceroprotein 1 (AQP1) transporter mutations or down-expression, or by drug efflux/sequestration through the concomitant overexpression of genes involved in the parasite thiol metabolism and of the gene encoding the ATP-binding cassette transporter ABC-C3 (MRPA) ${ }^{3,4}$ (Figure 1a). Other transporters of the $A B C$ superfamily also interfere with antimonials drug accumulation, including $A B C-C 7(P R P 1)^{5}$, $A B C-13^{6}, A B C-14^{7}$ or $A B C-G 2^{8}$. In addition, it has been shown that a number of intracellular proteins can also modulate the susceptibility of parasites to SbIII when they are overexpressed in experimental strains or clinical isolates. These include heat shock proteins (Hsp239, Hsp83 ${ }^{10}$ ), kinase $\left(\right.$ MAPK $1^{11}$ ), serine/threonine phosphatase (LinJ.12.0610 ${ }^{12}$ ) or proteins with still unknown functions $\left(\mathrm{ARM}^{\circ} 6^{9}, \mathrm{ARM}^{\circ} 8^{9}, \mathrm{P} 299^{13}\right)$. Overall, these results illustrate that Leishmania parasites have multiple strategies to acquire drug resistance and highlight the difficulty to define a conserved molecular marker of antimony resistance.

In a previous work ${ }^{14}$, we showed that Leishmania parasites are auxotrophic for NAD+ and rely on a salvage pathway to recycle NAD+ precursors from their environment (Figure 1a). The nicotinamidase PNC1 is a central enzyme of this pathway that controls NAD+ homeostasis by hydrolysing nicotinamide (NAm) to nicotinic acid (NA). Leishmania infantum parasites in which the pnc1 gene was disrupted ( $\Delta p n c 1)$ are depleted in NAD+ and cannot cause durable infections in mice, making of this enzyme an attractive target for drug development ${ }^{14,15}$. $\operatorname{NAD}(H)$, and its derived phosphorylated forms $N A D P(H)$, are essential cofactors required for 
72 energy-producing pathways and antioxidant defence in all living cells. They are further key

73 metabolites involved in host-pathogen interactions ${ }^{16}$. Here, we used NAD+ depleted $\Delta p n c 1$

74 mutant parasites to better understand SbIII mode of action and the mechanisms leading to

75 resistance to this drug. We found that intracellular NAD+ levels can directly modulate the

76 susceptibility of parasites to Sblll and identified the first mutation conferring antimony

77 tolerance and resistance in Leishmania. 


\section{Strains and cultures}

80

The pnc1 null mutant ( $\Delta p n c 1$ ) was previously generated by targeted gene replacement in the L. infantum (MHOM/MA/67/ITMAP-263) strain ${ }^{14}$. CRISPR/Cas9 genome edition experiments were performed in the L. major "Friedlin" reference strain (MHOM/IL/80/Friedlin, LEM 3171$)^{17}$. All strains and the derived mutants were maintained as promastigote forms in SDM79 medium supplemented with $10 \% \mathrm{FBS}$, penicillin/streptomycin and hemin $(5 \mathrm{mg} / \mathrm{L})$ (complete medium). Potassium antimonyl tartrate trihydrate (SbIII), L-Glutathione reduced (GSH), N-Acetyl-L-cysteine (NAC), nicotinic acid (NA) and MTT were all purchased from SigmaAldrich. Sblll-resistant parasites were generated by a stepwise approach starting with a drug concentration corresponding to the $\mathrm{EC}_{50}$ for that strain. Growth curve and $\mathrm{EC}_{50}$ were determined starting from an inoculum of $10^{6}$ parasites $/ \mathrm{mL}$ in $5 \mathrm{~mL}$ of complete medium, unless otherwise stated. Parasite density was determined daily (growth kinetics) or after 3 days of incubation with the drug ( $E C_{50}$ determination) using a flow cytometer ${ }^{14}$ or by measuring the OD at $600 \mathrm{~nm}$ (for graphical convenience, OD values were multiplied by 1000). All $\mathrm{EC}_{50}$ values were obtained with GraphPad Prism v5 software using a sigmoidal doseresponse model with variable slope. Cell viability assays on edited parasites were performed using a MTT test. In this assay, the yellow tetrazolium MTT dye was reduced to insoluble formazan crystals (purple color) in living cells using NADH as reducing agent. Briefly, $100 \mu \mathrm{l}$ of parasite cultures were mixed with $10 \mu \mathrm{L}$ of a MTT solution (10 mg/mL in PBS) and incubated $4 \mathrm{~h}$ at $26^{\circ} \mathrm{C}$. The reaction was stopped with $100 \mu \mathrm{L}$ of lysis solution ( $50 \%$ isopropanol/10\% SDS) and the plates were incubated for an additional $30 \mathrm{~min}$ with gentle shaking in the dark. The change in colour from yellow to purple was read at an absorbance of $600 \mathrm{~nm}$.

\section{$N A D(H) / N A D P(H)$ quantification}

Total (NAD+ plus NADH) or individual (NAD+ and NADH) dinucleotides were quantified using the bioluminescent NAD/NADH-Glo ${ }^{\mathrm{TM}}$ Assay (Promega) following manufacturer's instructions. This kit uses a proluciferin substrate to produce a light signal proportional to the amount of NAD and/or NADH present in the samples. Total (NADP+ plus NADPH) and individual (NADP+ and NADPH) phosphorylated forms were quantified similarly using the NADP/NADPH-Glo ${ }^{\text {TM }}$ 
Assay kit (Promega). Data are expressed as the mean value $( \pm S D)$ of Relative Luminescence Units (RLU) obtained for $10^{6}$ parasites and after 60 min of incubation.

\section{Quantitative RT-PCR analyses (qRT-PCR)}

All primers used in this study were designed with the primer3Plus software and are listed in table S1. Total RNA was extracted from parasite cultures in the exponential phase of growth using the RNeasy+ Mini Kit (Qiagen) with an additional treatment with turbo DNA-free DNase (Thermo Fischer Scientific). Two $\mu \mathrm{g}$ of total RNA was transcribed into complementary DNA (cDNA) using the Superscript reverse transcriptase III (Invitrogen) and oligo(dT)12-18 primers (Thermo Fischer Scientific), according to the manufacturer's instructions. Diluted cDNA (1/10) was used for the qPCR reactions in $10 \mu \mathrm{L}$ final volume with SYBR Green I Master on a LightCycler480 (Roche). The relative expression of each gene was determined from two biological replicates with the $2^{-\triangle \triangle C T}$ method and the GAPDH gene as reference, using the LightCycler480 software.

\section{CRISPR/Cas9 genome editing and off-target mutation analysis}

The marker-free nucleotide editing approach developed in P. Falciparum ${ }^{18}$ was adapted to introduce the H451Y mutation in the L. major reference strain. The 20nt single guide RNA (sgRNA) sequence TTTGATCGACTCCGAGCACT (the targeted nucleotide is underlined) was based on the LmjF.33.1710 orthologue gene sequence. Plasmids were constructed as described in ${ }^{17}$. Briefly, a donor DNA sequence that consists of an $880 \mathrm{bp}$-long intragenic region of LmjF.33.1710 gene was generated by PCR fusion using the modified primer pairs Lm1F/Lm1R and Lm2F/Lm2R (Table S1) to create the C1351T mutation and a "shield" silent mutation (G1356T) in the protospacer adjacent motif (PAM) to prevent further Cas9-mediated cleavage (Figure 2f). The donor DNA sequence was cloned in the pLS5 plasmid digested with $K p n I$ and $X b a l$. To obtain the 20 nt sgRNA sequence surrounded by the $15 \mathrm{nt}$ adaptors necessary for In-Fusion ${ }^{\circledR}$ cloning, the two oligonucleotides Seed_F and Seed_R were annealed by incubation in boiling water for $2 \mathrm{~min}$ followed by gentle cooling in room-temperature water for $2 \mathrm{~h}$. The sgRNA sequence and adaptors were then cloned in the Bsgl-digested pLS5-donor DNA plasmid using the In-Fusion HD cloning kit (Clonetech). pLS5-donor DNA plasmids with and without the sgRNA sequence were transfected in the L. major Friedlin strain that harbours the pTCAS9 plasmid $^{17}$ to generate edited (Lm_H451Y) and control (Lm_ctrl) cell lines, 
respectively. After transfection and selection with hygromycin and puromycin, gene edition was checked by PCR amplification and Sanger sequencing using the LmiSCAMK_F/LmiSCAMK_R primer pair (Table S1). Once heterozygosity was confirmed in the mutated locus, parasites were cloned by limiting dilution. A parasite clone containing the homozygous desired and shield mutations (named Lm_H451Y) was selected for subsequent phenotype analyses. To confirm the absence of off-target mutations, the genomes of Lm_ctrl and Lm_H451Y strains were sequenced (see below) and off-target candidates (up to five mismatches allowed) were identified using the Protospacer Workbench software suite ${ }^{19}$. For each candidate, the vicinity (within a 21 nt window) to specific INDELs of the edited cell lines was assessed as described in Vasquez et al. ${ }^{20}$ (Table S2).

\section{WGS and analyses}

Parasite genomic DNA was extracted using the QIAamp DNA Mini Kit (Qiagen). A paired-end sequencing library for $\Delta p n c 1$ parasites was prepared with the Nextera DNA Sample Preparation Kit (Illumina) and sequenced on an Illumina HiSeq 2500 (2×250 bp) apparatus. DNA samples from the KO-SbR, Lm_ctrl, Lm_H451Y and Lm_H451Y-SbR strains were sheared using a Bioruptor Pico sonication device (Diagenode) to yield $\approx 400$ bp fragments. Libraries were constructed for WGS as previously described ${ }^{21}$. Paired-end sequencing ( $2 \times 150 \mathrm{bp}$ ) was performed on an Illumina MiSeq apparatus with the MiSeq Reagent Kit V2. The presence of SNPs and INDELs was determined using the EuPathDB Galaxy platform ${ }^{22}$ and the workflow pipeline for "Variant Calling, paired-end sequencing" and pre-loaded reference genomes for the L. infantum JPCM5 and L. major Friedlin strains (TritrypDB build 29). Filtered Variant Call Format (VCF) files corresponding to SNPs and INDELs with an impact on coding sequences were compared between the $\Delta p n c 1$ and KO-SbR libraries. Specific SNPs/INDELs detected in KO-SbR (16 SNPs and 15 INDELs) were manually verified with the IGV 2.4.8 visualization tool, and eliminated if already present in the $\Delta p n c 1$ parental line. After this verification step, only three specific SNPs were retained for analysis (Table S3). The same strategy was used to compare SNPs/INDELs in the Lm_ctrl, Lm_H451Y and Lm_H451Y-SbR libraries and only SNPs and INDELS with read depth $>2$ were kept for further analysis (Table S4). The CNV-seq pipeline was used to identify CNVs potentially associated with drug resistance ${ }^{23}$. This pipeline identifies localized regions in which the read depth normalized across the length of the chromosome differs significantly between samples. For $\Delta p n c 1$ and KO-SbR pairwise comparisons, a $3 \mathrm{~kb}$ 
168 window size was chosen, but similar results were obtained with smaller and larger window 169 sizes (Table S5). For Lm_H451Y and Lm_H451Y-SbR pairwise comparisons, the sliding window 170 size was automatically determined (4265 bp). Input hits files were derived from the Binary 171 Alignment Map (BAM) files generated by the EupathDB Variant Calling pipeline.

\section{Accession number}

173 All sequencing data have been submitted to the European Nucleotide Archive (ENA) and are 174 available under the accession number PRJEB27329. 


\section{Apnc1 parasites are highly susceptible to SbIII}

177 When grown in SDM79 medium, $\Delta p n c 1$ promastigotes had similar growth rates than wild type 178 (WT) parasites (Figure 1b). However, they are about ten-fold more susceptible to SbIII (EC 50 $179 \Delta p n c 1=2.51 \mu \mathrm{g} / \mathrm{mL} ; E_{50} \mathrm{WT}=20.92 \mu \mathrm{g} / \mathrm{mL}$ ) (Figure 1c). This phenotype could be rescued by NA supplementation in the growth medium (Figure 1c). NA supplementation also induced a dose-dependent increase of both $\operatorname{NAD}(\mathrm{H})$ and $\operatorname{NADP}(\mathrm{H})$ content in $\Delta p n c 1$ parasites where these pools are significantly depleted (Figure 1d). Conversely, it did not have any effect on $\operatorname{NAD}(\mathrm{H})$ and $\operatorname{NADP}(\mathrm{H})$ content in WT parasites (Figure 1d). Individual dinucleotides measurements showed that $\triangle p n c 1$ parasites were specifically depleted in NAD+ and NADPH forms (Figure 1e). As the Leishmania redox homeostasis is also maintained by de novo biosynthesis of reduced trypanothione (Figure 1a), we tested whether supplementation with trypanothione precursors could restore the physiological Sblll susceptibility in $\Delta p n c 1$ parasites. Supplementation with $\mathrm{N}$-acetyl-cysteine (NAC) or reduced glutathione (GSH) decreased the susceptibility of $\Delta p n c 1$ parasites to SbIII (Figure 1f). Altogether, these results established a direct connection between Leishmania NAD+ metabolism, SbIII toxicity and thiol metabolism (Figure 1a). qRT-PCR assays further indicated that known genes involved in SbIII mode of action and resistance were similarly transcribed in $\Delta p n c 1$ and WT parasites, except for GSH1, the rate-limiting step in glutathione biosynthesis, for which the transcript level was increased 2-fold in $\Delta p n c 1$ (Figure 1g).

$\triangle p n c 1$ parasites resistant to SbIII show a single amino acid substitution in the SCAMK gene

To better understand the link between NAD+ homeostasis and SbIII susceptibility, we tried to generate Sblll-resistant WT and $\Delta p n c 1$ parasites (named WT-SbR and KO-SbR, respectively) by stepwise selection in the presence of increasing concentrations of the drug. We could select KO-SbR parasites that proliferated in the presence of up to $320 \mu \mathrm{g} / \mathrm{mL}$ of SbIII $\left(E C_{50}=309 \mu \mathrm{g} / \mathrm{mL}\right)$, but not at higher concentrations (Figure 2a). In WT-SbR parasites (EC 50 $>1000 \mu \mathrm{g} / \mathrm{mL}$ ), MRPA was ten-fold upregulated as compared with WT parasites (Figure $2 \mathrm{~b}$ ) which is in agreement with the known model of antimony resistance ${ }^{3,4}$ and the presence of an extrachromosomal amplicon of $15 \mathrm{~kb}$ bearing MRPA gene in the WT-SbR strain (data not shown). Conversely, qRT-PCR analysis showed a modest, but paradoxical overexpression of 
the genes encoding the AQP1 transporter and MRPA efflux-pump in KO-SbR parasites (Figure $2 b)$. Moreover, the levels of NAD+, NADH, NADP+ and NADPH were similar in KO-SbR and $\Delta$ pnc1 parasites (Figure 2c). As drug resistance in Leishmania is often associated with gene copy number variations (CNVs) or SNPs ${ }^{24}$, we sequenced the genomes of $\Delta p n c 1$ and KO-SbR parasites by Illumina paired-end sequencing. As expected, no read aligned to the pnc1 gene, confirming its deletion in the both strains (Figure S1). All CNVs detected in KO-SbR mutants were located in non-coding telomeric or intergenic regions (Table S5). The look for nonsynonymous SNPs only present in the KO-SbR strain identified a single homozygous mutation (C1351T) leading to the H451Y substitution in a putative protein kinase gene (LinJ.33.1810) (Table S3). The fixation of the H451Y mutation in KO-SbR parasites was confirmed by PCR and Sanger sequencing (Figure 2d). Interestingly, another point mutation (E629K) in the Cterminus of the LinJ.33.1810 gene was already described in a L. infantum SbIII-resistant strain bearing MRPA amplification ${ }^{25}$. This single copy gene defines a new kinase family recently named SCAMK ${ }^{26}$ that is specific to Metakinetoplastina protists and functionally related to the calcium-dependent protein kinases (CDPKs) present in plants and protozoans ${ }^{27}$. CDPKs have been widely studied in apicomplexan parasites where they are considered to be important signal transducers involved in parasite motility, invasion or host cell egress ${ }^{28}$. Specifically, $\mathrm{Ca}^{2+}$ binding to the EF-hand domains of CDPKs induces a global conformational change of the protein, resulting in the activation of the kinase activity. The $\mathrm{H}$ residue in position 451 , which is mutated in the KO-SbR strain, is located within the calcium-binding site of the third EF-hand domain of Leishmania SCAMK, and is conserved across kinetoplastids (Figure 2e).

\section{The H451Y mutation generates SbIII tolerant Leishmania parasites}

To gain insight into the role of the H451Y mutation in SbllI resistance, we used the CRISPR/cas9 genome editing technology ${ }^{17}$ with a marker-free nucleotide editing approach ${ }^{18}$ to introduce the H451Y mutation in the Leishmania major WT "Friedlin" reference strain. $L$. major parasites expressing Cas9 from an episomal plasmid were transfected with a second plasmid bearing an sgRNA complementary to the L. major SCAMK orthologue (LmjF.33.1710), and a donor sequence including the desired mutation (C1351T) and a shield mutation (G1356T) in the PAM motif (Lm_H451Y strain) (Figure 2f). As a control, we transfected parasites with the same plasmid backbone, but without the sgRNA (Lm_ctrl strain) (Figure 2g). After selection and cloning, we confirmed the presence of the $\mathrm{H} 451 \mathrm{Y}$ mutation (Figure $2 \mathrm{~g}$ ) and 
the absence of off-target mutations (Table S2) by Sanger and WGS, respectively. Phenotype analyses indicated that Lm_ctrl and Lm_H451Y strains had similar growth rates (Figure 3a). The Sblll susceptibility of both strains was compared using a MTT-based assay test and an inoculum of $10^{6}$ parasites $/ \mathrm{mL}$. As shown in figure $3 \mathrm{~b}, \mathrm{Lm} \_\mathrm{H} 451 \mathrm{Y}$ parasites are not more resistant than controls. Surprisingly, Lm_H451Y (but not Lm_ctrl) parasites exposed to the highest drug concentrations resumed growth after seven days in culture (Figure 3c). Analysis of the growth curves obtained starting from a higher parasite inoculum $\left(4.10^{6}\right.$ parasites $\left./ \mathrm{mL}\right)$ and using cytocidal concentrations of SbIII showed that Lm_H451Y parasites can survive and slowly proliferate in the presence of $1000 \mu \mathrm{g} / \mathrm{mL}$ of SbIII, which is the limit of SbIll solubility in the culture medium (Figure 3d). In a parallel experiment, the viability of edited parasites exposed to maximal Sblll concentrations has been confirmed and quantified by MTT-based assays performed at days 3 and 5 of culture (Figure 3e). Light-microscopy examination after exposure to the maximal concentration of Sblll revealed the presence of a heterogenous population of dead and unstressed promastigote forms in Lm_H451Y cultures, and the absence of surviving parasites in Lm_ctrl cultures (Figures $3 f$ and S2). In agreement, we detected intact high molecular weight genomic DNA in all tested conditions for Lm_H451Y parasites, but only up to $250 \mu \mathrm{g} / \mathrm{mL}$ of SbIII for Lm_ctrl parasites (Figure 3g). The ability of a microorganism to survive lethal concentrations of a drug while keeping similar $\mathrm{EC}_{50}$ than susceptible strains is characteristic of drug tolerant/persistent microorganisms ${ }^{29}$. This phenomenon is known to facilitate drug resistance acquisition in bacteria but remains largely understudied in protozoan parasites ${ }^{30}$. To confirm the role of the H451Y mutation in Leishmania SbIII tolerance, we transiently exposed parasites to various bolus doses of SbIII for two days, and followed their growth after drug withdrawal. The growth of Lm_ctrl parasites was delayed and they could survive after transient exposure to Sblll concentrations up to 400 $\mu \mathrm{g} / \mathrm{mL}$ (Figure 3h). Conversely, Lm_H451Y parasites recovered within 10 days post-bolus, whatever the concentration of Sblll used (Figure 3h). The toxicity of most anti-leishmanial drugs has been partly attributed to induction of reactive oxygen species (ROS) formation and consequently to oxidative damage. Therefore, we carried out similar growth curve experiments in the presence of miltefosine, amphotericin $B$, the oxidative stress inducer menadione, or $\mathrm{H}_{2} \mathrm{O}_{2}$ (Figure S3). We did not detect any difference between Lm_H451Y and Lm_ctrl parasites in all tested conditions, suggesting that the tolerance phenotype induced by 
268 to oxidative stress. Next, we tested whether Lm_H451Y parasites were prone to rapidly 269 develop resistance to SbIII. As expected, highly resistant Lm_H451Y parasites (named 270 Lm_H451Y-SbR) were selected after only five passages (P5) in the presence of $1000 \mu \mathrm{g} / \mathrm{mL}$ of 271 SbIII ( $E C_{50}>1000 \mu \mathrm{g} / \mathrm{mL}$ ) and this resistance phenotype remained stable up to P15 (Figure 3i).

272 Genome sequencing of Lm_H451Y-SbR parasites cultured for 10 passages in the presence of $2731000 \mu \mathrm{g} / \mathrm{mL}$ of SbIII did not reveal any new CNV compared with the Lm_H451Y parental cell 274 line. Moreover, non-synonymous SNPs specific to Lm_H451Y-SbR were all heterozygous and 275 only concerned hypothetical proteins or factors not known to be involved in SbIll resistance 276 (Table S4). These results corroborate our initial observations in the L. infantum KO-SbR mutant 277 and show that the H451Y mutation is necessary and sufficient to generate SbllI-tolerant 278 parasites that can rapidly evolve into resistant parasites in the presence of drug pressure. 
Drug resistance in eukaryotic microorganisms shares many similarities with antibiotic resistance in bacteria ${ }^{3}$. Our knowledge about drug resistance mechanisms in Leishmania (and other protozoan parasites) mainly results from experiments using growth inhibitory measurements that determine the lowest drug concentration needed to inhibits parasite growth by $50 \%\left(\mathrm{EC}_{50}\right)$. Mechanisms of drug action and resistance patterns can be however different when measured at dosages that kill the parasite, not just inhibit its growth ${ }^{31}$. Moreover, these approaches do not allow the identification of parasite subpopulations with variable levels of resistance. In bacteria or fungi, drug tolerant subpopulations (also known as "persisters") can survive at lethal drug concentrations while being still sensitive to cytostatic effect of the drug. The mechanisms leading to persisters formation are diverse and can include genetic mutations that increase the proportion of persister cells within a population ${ }^{32,33}$. Importantly, persisters are known to cause treatment failure due to relapsing infections and behave as an evolutionary reservoir of drug resistance ${ }^{30,34,35}$. The phenomenon of drug tolerance/persistence in protozoan parasites and its link with parasite drug resistance and treatment failure have been poorly studied to date. In trypanosomatids, the ability of drugsensitive parasites to survive a lethal and prolonged drug exposure has been recently described in Trypanosoma $\mathrm{cruzi}^{36}$ and Leishmania ${ }^{37}$. However, these phenotypes have been attributed to clearly distinct mechanisms involving a metabolic dormancy state and a genetic preadaptation to resistance, respectively.

In this study, we report that (i) Leishmania NAD+ depletion led to SbIII hypersusceptibility that is rescued by NAD+ precursor or trypanothione precursors supplementation; (ii) NAD+ depleted parasites can develop SbIII resistance through a single amino acid substitution in a metakinetoplastid-specific kinase; (iii) this mutation makes Leishmania parasites tolerant to high concentrations of SbIII and facilitates the emergence of resistant parasites without additional genomic modification. Although these findings have been obtained in mutants selected under laboratory conditions in vitro, they provide novel insights into the mode of action of antimony and establish that the orphan Leishmania kinase SCAMK is a new central actor of Sblll mode of action and resistance. Because the H451Y mutation is precisely located within the calcium-binding site of the third EF-hand domain of Leishmania SCAMK, we can speculate that it should directly impair $\mathrm{Ca}^{2+}$ binding and the associated conformational change 
310 required for the enzymatic activation of CDPKs. The study of the structural consequences of 311 the H451Y mutation should help us in understanding how this orphan kinase integrates 312 calcium signaling to control the lethal effect of SbIll and could result in new therapeutic 313 applications. We were unable to analyse the tolerance phenotype of edited parasites in the 314 intracellular amastigote stage because the L. major strain used in this study is a reference 315 strain that lost its virulence due to repeated passages in vitro. Further work is therefore 316 required to determine whether this mutation can lead to treatment failure and persistent 317 infection in vivo and to assess whether it is harmful or whether it can be spread in natural 318 populations. Nevertheless, the correlation between Leishmania SCAMK gene polymorphisms 319 and antimony treatment failure in clinical isolates could constitute the first step towards the 320 identification of a new molecular marker of antimony tolerance/resistance in the field. 
321 Acknowledgements: We thank the MGX platform (Montpellier, France) and Dr Mallorie Hide 322 for HiSeq sequencing, and the UMR DIADE (Dynadiv team) for library preparation and MiSeq 323 sequencing at CIRAD (Montpellier, France). We are also thankful to Elisabetta Andermarcher 324 for assistance in editing the manuscript.

325 Funding: This work was supported by the Institut de Recherche pour le Développement (IRD), 326 the Centre National de la Recherche Scientifique (CNRS), the French Ministry of Research and 327 the Centre Hospitalier Universitaire of Montpellier institutional fundings. LS, JJLR, and YS were 328 supported by the Laboratoire d'Excellence (LabEx) ParaFrap (the French Parasitology Alliance 329 for Health Care, grant number ANR-11- LABX-0024). 
331 1. Burza S, Croft SL, Boelaert M. Leishmaniasis. The Lancet 2018; 392: 951-70.

332 2. Wyllie S. Dual action of antimonial drugs on thiol redox metabolism in the human pathogen 333 Leishmania donovani. J Biol Chem 2004; 279: 39925-32.

334 3. Fairlamb AH, Gow NAR, Matthews KR et al. Drug resistance in eukaryotic microorganisms. Nat Microbiol 2016; 1: 16092.

4. Ponte-Sucre A, Gamarro F, Dujardin J-C et al. Drug resistance and treatment failure in leishmaniasis: A 21st century challenge. PLoS Negl Trop Dis 2017; 11: e0006052-24.

338 5. Leprohon $P$, Légaré $D$, Ouellette $M$. Intracellular localization of the $A B C C$ proteins of Leishmania and their role in resistance to antimonials. Antimicrob Agents Chemother 2009; 53: 2646-9.

6. Arcari T, Manzano JI, Gamarro F. $A B C l 3$ is a new mitochondrial $A B C$ transporter from Leishmania major involved in susceptibility to antimonials and infectivity. Antimicrob Agents Chemother 2017; 61: 113-8.

7. Manzano JI, Garcia-Hernandez R, Castanys S et al. A new ABC half-transporter in Leishmania major is involved in resistance to antimony. Antimicrob Agents Chemother 2013; 57: 3719-30.

8. Perea A, Manzano JI, Castanys S et al. The LABCG2 transporter from the protozoan parasite Leishmania is involved in antimony resistance. Antimicrob Agents Chemother 2016; 60: 348996.

9. Tejera Nevado $\mathrm{P}$, Bifeld E, Höhn $\mathrm{K}$ et al. A telomeric cluster of antimony resistance genes on chromosome 34 of Leishmania infantum. Antimicrob Agents Chemother 2016; 60: 5262-75.

10. Vergnes B, Gourbal B, Girard I et al. A proteomics screen implicates HSP83 and a small

352 kinetoplastid calpain-related protein in drug resistance in Leishmania donovani clinical field isolates by modulating drug-induced programmed cell death. Mol Cell Proteomics 2007; 6: 88354101. 
11. Garg M, Goyal N. MAPK1 of Leishmania donovani modulates antimony susceptibility by downregulating P-glycoprotein efflux pumps. Antimicrob Agents Chemother 2015; 59: 385363.

12. Gazanion E, Fernandez-Prada C, Papadopoulou B et al. Cos-Seq for high-throughput identification of drug target and resistance mechanisms in the protozoan parasite Leishmania. Proc Natl Acad Sci USA 2016; 113: E3012-21.

13. Choudhury $\mathrm{K}$, Zander $\mathrm{D}$, Kube $\mathrm{M}$ et al. Identification of a Leishmania infantum gene mediating resistance to miltefosine and SbIII. Int J Parasitol 2008; 38: 1411-23.

14. Gazanion E, Garcia D, Silvestre R et al. The Leishmania nicotinamidase is essential for NAD(+) production and parasite proliferation. Mol Microbiol 2011; 82: 21-38.

15. Michels PAM, Avilán L. The NAD(+) metabolism of Leishmania, notably the enzyme nicotinamidase involved in $\mathrm{NAD}(+)$ salvage, offers prospects for development of anti-parasite chemotherapy. Mol Microbiol 2011; 82: 4-8.

16. Mesquita I, Varela $P$, Belinha $A$ et al. Exploring $N A D(+)$ metabolism in host-pathogen interactions. Cell Mol Life Sci 2016; 73: 1225-36.

17. Sollelis L, Ghorbal M, MacPherson CR et al. First efficient CRISPR-Cas9-mediated genome editing in Leishmania parasites. Cell Microbiol 2015; 17: 1405-12.

18. Ghorbal M, Gorman M, MacPherson CR et al. Genome editing in the human malaria parasite Plasmodium falciparum using the CRISPR-Cas9 system. Nat Biotechnol 2014; 32: 81921.

19. MacPherson CR, Scherf A. Flexible guide-RNA design for CRISPR applications using Protospacer Workbench. Nat Biotechnol 2015; 33: 805-6.

20. Vasquez JJ, Wedel C, Cosentino RO et al. Exploiting CRISPR-Cas9 technology to investigate individual histone modifications. Nucleic Acids Res 2018; 46: e106. 
21. Mariac C, Scarcelli N, Pouzadou J et al. Cost-effective enrichment hybridization capture of chloroplast genomes at deep multiplexing levels for population genetics and phylogeography studies. Mol Ecol Resour 2014; 14: 1103-13.

22. Aurrecoechea $C$, Barreto $A$, Basenko EY et al. EuPathDB: the eukaryotic pathogen genomics database resource. Nucleic Acids Res 2017; 45: D581-91.

23. Xie C, Tammi MT. CNV-seq, a new method to detect copy number variation using highthroughput sequencing. BMC Bioinformatics 2009; 10: 80-9.

24. Leprohon P, Fernandez-Prada C, Gazanion E et al. Drug resistance analysis by next generation sequencing in Leishmania. Int J Parasitol Drugs Drug Resist 2015; 5: 26-35.

25. Brotherton MC, Bourassa S, Leprohon $P$ et al. Proteomic and genomic analyses of antimony resistant Leishmania infantum mutant. PLOS ONE 2013; 8: e81899.

26. Chen F, Zhang L, Lin Z et al. Identification of a novel fused gene family implicates convergent evolution in eukaryotic calcium signaling. BMC Genomics 2018; 19: 306.

27. Harper JF, Harmon A. Plants, symbiosis and parasites: a calcium signalling connection. Nat Rev Mol Cell Biol 2005; 6: 555-66.

28. Billker O, Lourido S, Sibley LD. Calcium-dependent signaling and kinases in apicomplexan parasites. Cell Host Microbe 2009; 5: 612-22.

29. Fisher RA, Gollan B, Helaine S. Persistent bacterial infections and persister cells. Nat Rev Microbiol 2017; 15: 453-64.

30. Cohen NR, Lobritz MA, Collins JJ. Microbial persistence and the road to drug resistance. Cell Host Microbe 2013; 13: 632-42.

31. Roepe PD. To kill or not to kill, that is the question: cytocidal antimalarial drug resistance. Trends Parasitol 2014; 30: 130-5.

32. Michiels JE, Van den Bergh $\mathrm{B}$, Verstraeten $\mathrm{N}$ et al. Molecular mechanisms and clinical implications of bacterial persistence. Drug Resist Updat 2016; 29: 76-89. 
404 33. Balaban NQ, Helaine $\mathrm{S}$, Lewis $\mathrm{K}$ et al. Definitions and guidelines for research on antibiotic 405 persistence. Nat Rev Microbiol 2019; doi: 10.1038/s41579-019-0196-3. [Epub ahead of print] 406

407 34. Delarze E, Sanglard D. Defining the frontiers between antifungal resistance, tolerance and 408 the concept of persistence. Drug Resist Updat 2015; 23: 12-9.

409 35. Levin-Reisman I, Ronin I, Gefen O et al. Antibiotic tolerance facilitates the evolution of 410 resistance. Science 2017; 355: 826-30.

411 36. Sánchez-Valdéz FJ, Padilla A, Wang W et al. Spontaneous dormancy protects Trypanosoma 412 cruzi during extended drug exposure. eLife 2018; 7: 833.

413 37. Dumetz F, Cuypers B, Imamura H et al. Molecular preadaptation to antimony resistance in 414 Leishmania donovani on the Indian subcontinent. mSphere 2018; 3: e00548-17. 
Figure 1. $\Delta$ pnc1 parasites are highly susceptible to SbIII. (a) Schematic representation of the cross-talk between Leishmania NAD+ metabolism, trivalent antimony (SbIII) mode of action and thiol metabolism. NA, nicotinic acid; NAm, nicotinamide; NR, nicotinamide riboside; NAMN, nicotinic acid mononucleotide; NAAD, nicotinic acid dinucleotide; NADK, NAD+ kinase; PPP, pentose phosphate pathway; G6PDH, glucose-6-phosphate dehydrogenase; AQP1, aquaglyceroporin 1; TR, trypanothione reductase; $\mathrm{T}(\mathrm{SH}) 2$, reduced trypanothione; $\mathrm{T}(\mathrm{S}) 2$, oxidized trypanothione; TXN1, tryparedoxin 1; MRPA, ABCC3 transporter; Glu, glutamate; Cys, cysteine; Orn, ornithine; GSH1, gamma-glutamylcysteine synthetase; GSH, glutathione; Spd, spermidine; GSpdS, glutathionylspermidine synthetase; Gspd, glutathionylspermidine; TryS, trypanothione synthase. (b) Growth curves of L. infantum WT and $\Delta p n c 1$ promastigotes in SDM79 medium supplemented with NA (1, 10 and $100 \mu \mathrm{M})$. Data are representative of three independent experiments. (c) Effect of Sblll on the growth kinetics of L. infantum WT and $\Delta p n c 1$ parasites (supplemented or not with 1, 10 and $100 \mu \mathrm{M} N \mathrm{~A}$ ). Data are the mean \pm SD of two biological replicates and are representative of at least five independent experiments. (d) Intracellular NAD(H) (left) and NADP(H) (right) levels measured in L. infantum WT (black bars) and $\Delta p n c 1$ (white bars) parasites after 2 days of culture in the presence of increasing concentrations of NA (quantification of parallel series of cultures as described in Methods section). Values are expressed as Relative Luminescence Units (RLU) for $10^{6}$ parasites and are the mean $\pm S D$ of triplicate measurements. (e) Relative quantification of NAD+, NADH, NADP+ and NADPH in WT and $\triangle p n c 1$ parasites after 2 days of growth. Data are the mean \pm SD of duplicate measurements and are representative of two independent experiments. (f) Effect of GSH and N-acetyl cysteine (NAC) supplementation on the growth kinetics of L. infantum WT and $\Delta p n c 1$ parasites in the presence of increasing concentrations of SbIII. Data are the mean \pm SD of duplicate measurements and are representative of three independent experiments. (g) Relative gene expression levels of candidate genes involved in Sblll mode of action/resistance in $\Delta p n c 1$ parasites relative to the WT strain (see above for abbreviations). The fold-change cut-offs ( 0.5 and 1.5$)$ are represented by dashed lines. 
the presence of increasing concentrations of Sblll was monitored at $72 \mathrm{~h}$ by measuring the culture $\mathrm{OD}$ at $600 \mathrm{~nm}$. Data are the mean \pm SD of duplicate measurements and are representative of three independent experiments. (b) Relative quantification of transcript abundance in WT-SbR relative to WT parasites (left panel) and in KO-SbR relative to $\Delta p n c 1$ parasites (right panel). The fold-change cut-offs ( 0.5 and 1.5 ) are represented by dashed lines. (c) Quantification of NAD+, NADH, NADP+ and NADPH levels in KO-SbR parasites relative to $\Delta p n c 1$ parental line after 2 days of growth. Dotted line, ratio $=1$. Data are the mean \pm SD RLU ratio values obtained from two biological replicates, each measured in duplicate. (d) Comparison of the LinJ.33.1810 gene sequences in $\Delta p n c 1$ (top) and KO-SbR (bottom) parasites showing the $\mathrm{C}$ to T substitution (asterisk) in KO-SbR parasites. (e) Schematic representation of the functional domains present in the protein encoded by the LinJ.33.1810 gene using the ScanProsite tool (https://prosite.expasy.org/scanprosite/). Lower panel: alignment of the EFhand domain 3 from LinJ.33.1810 orthologues in L. major (LmjF.33.1710), L. donovani (LdBPK_331810.1), L. braziliensis (LbrM.33.1980), Trypanosoma brucei (Tb927.2.1820) and Trypanosoma cruzi (TCCLB.510257.130). The black arrow indicates the conserved $\mathrm{H}$ residue in position 451. The calcium-binding site of EF hand 3 (EF-loop 3) is underlined. (f) Position of the sgRNA sequence and the protospacer adjacent motif (PAM) in the LmjF.33.1710 sequence used to introduce the H451Y mutation in L. major with the CRISPR/Cas9 gene editing system. The partial donor DNA sequence illustrates the presence of the targeted and shield mutations. (g) Left: schematic representation of the plasmids used to generate the Lm_ctrl and Lm_H451Y strains. Right: chromatograms corresponding to part of the LmjF.33.1710 gene sequence (Sanger method). The targeted and shield mutations are indicated by a red and a green asterisk, respectively.

Figure 3. The H451Y mutation generates SbIII tolerant and resistant Leishmania parasites. (a) Growth curves of Lm_ctrl and Lm_H451Y parasites in SDM79 medium. Data are the mean \pm SD of two biological replicates and are representative of at least three independent experiments. (b) Sblll susceptibility of Lm_ctrl and Lm_H451Y parasites. Growth in the presence of increasing concentrations of SbIII $(25,50,100,200,400,600 \mu \mathrm{g} / \mathrm{mL})$ was monitored at $72 \mathrm{~h}$ by MTT-based assay. Data are the mean \pm SD of two biological replicates and representative of two independent experiments. (c) Same growth curves experiments as in (b) showing that Lm_H451Y parasites exposed to the highest SbIII concentrations restart to 
476 growth after 7 days. (d) Growth curves of Lm-ctrl (left) and Lm_H451Y (right) parasites seeded

477 at $4.10^{6}$ parasites $/ \mathrm{mL}$ and exposed to cytocidal concentrations of SbIII $(250,500$ and 1000

$478 \mu \mathrm{g} / \mathrm{mL}$ ) for eight days. Data are the mean \pm SD of duplicate OD measurements and are 479 representative of three independent experiments. (e) Quantification of Lm_ctrl and 480 Lm_H451Y parasites viability using MTT-based assays. Parasites were seeded at $4.10^{6}$ 481 parasites $/ \mathrm{mL}$ and exposed to high concentrations of Sblll $(100,250,500,1000 \mu \mathrm{g} / \mathrm{ml})$ for 3 482 and 5 days. Data are expressed as OD $600 \mathrm{~nm}$ values and are the mean \pm SD of two biological 483 replicates. (f) Light-microscopy images of Lm_ctrl (top panel) and Lm_H451Y (bottom panel) 484 parasites exposed to $1000 \mu \mathrm{g} / \mathrm{mL}$ of Sblll for 3 days (EVOS FL inverted microscope and x20 485 magnification). (g) Integrity of genomic DNA extracted from $5 \mathrm{~mL}$ of parasite cultures seeded 486 at $4.10^{6}$ parasites $/ \mathrm{mL}$ after 3 days of culture in the presence of different SbIII concentrations $487(0,250,500$ and $1000 \mu \mathrm{g} / \mathrm{mL}$ ). MW: GeneRuler 1kb DNA ladder. (h) Lm_ctrl (left) and 488 Lm_H451Y (right) parasites were incubated with increasing concentrations of SbIII (100 to 800 $489 \mu \mathrm{g} / \mathrm{mL}$ ) for $48 \mathrm{~h}$. Parasites were then centrifuged, washed three times with PBS and grown in 490 drug-free medium for ten days. The experiment timeline is shown at the bottom. Parasite 491 density (OD) was checked daily. Data are the mean \pm SD of two duplicate measurements and 492 are representative of two independent experiments. (i) Growth inhibition assay of Lm_H451Y 493 parasites cultured with $1000 \mu \mathrm{g} / \mathrm{mL}$ SbIII for 1 (P1), 5 (P5), 10 (P10) and 15 (P15) consecutive 494 passages. 


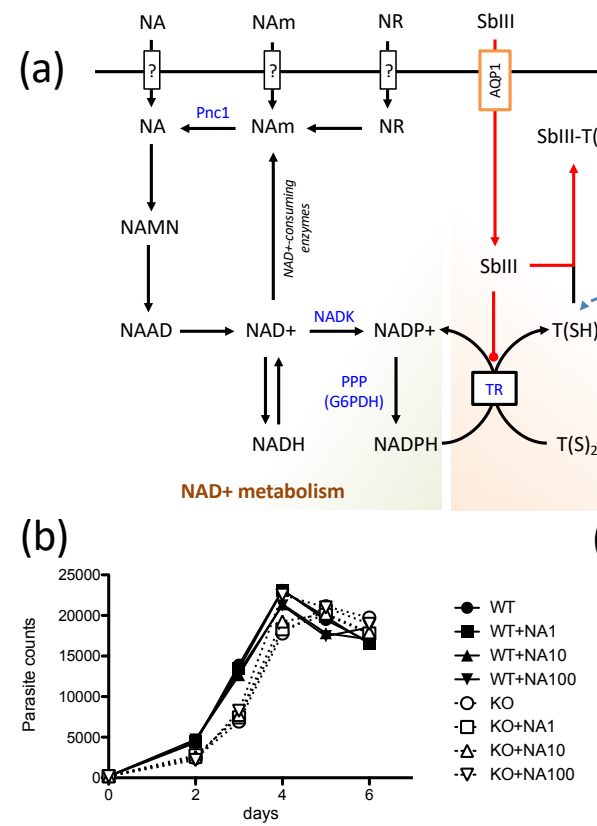

(d)

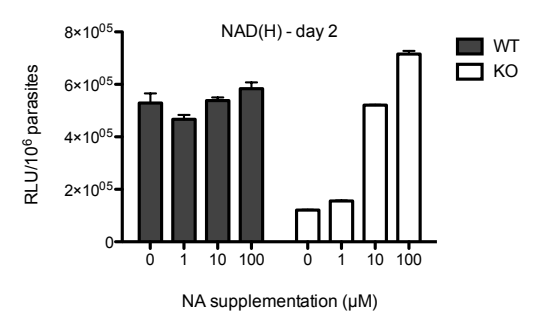

(e)

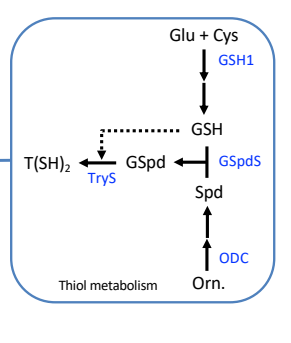

SbIII mode of action

(c)

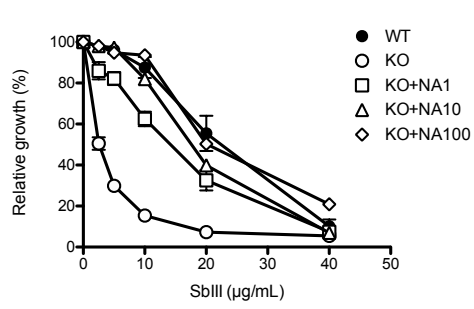

(f)
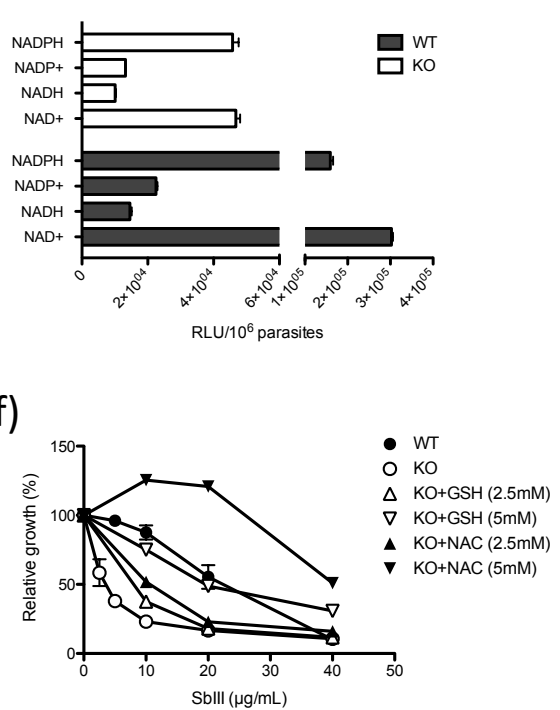

(g)
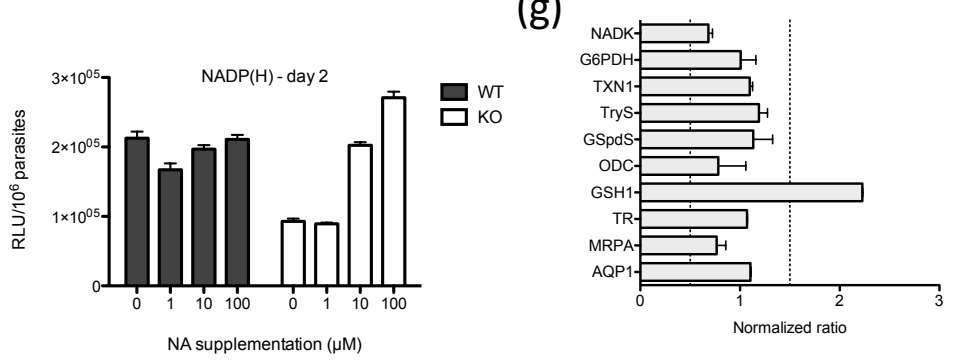

Figure 1 
(a)

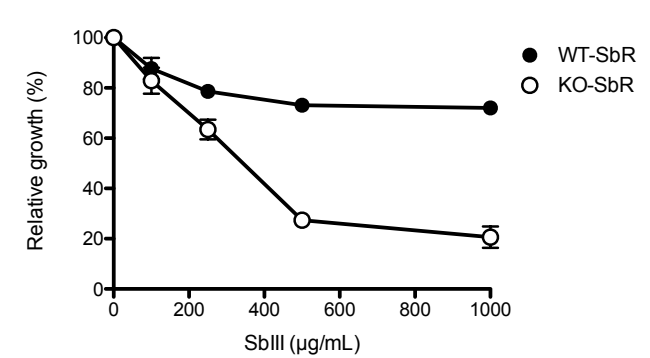

(c)

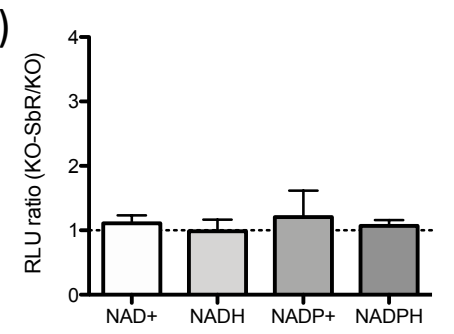

(d)

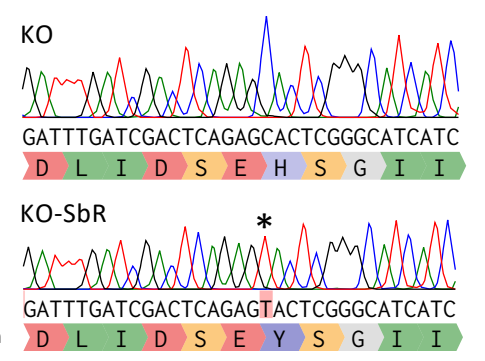

(b)

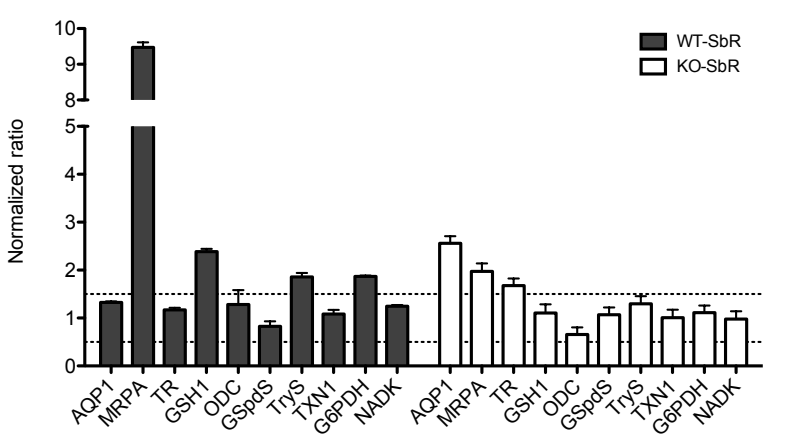

(e)

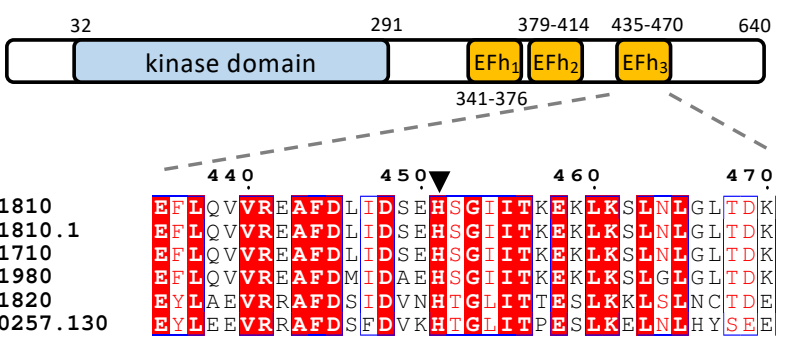

(f)

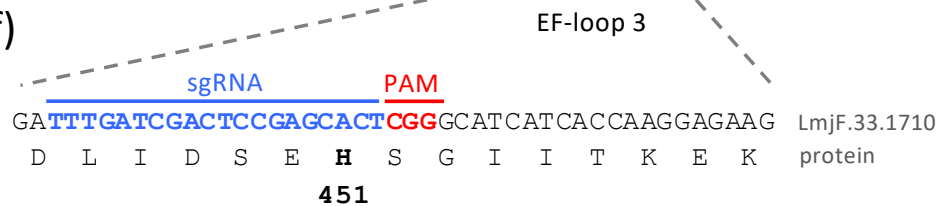

GATTTGATCGACTCCGAGTACTCTGGCATCATCACCAAGGAGAAG Donor DNA

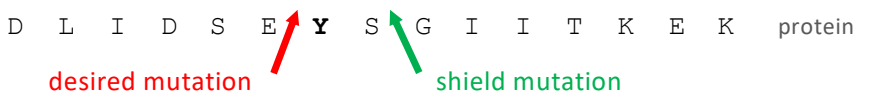

(g)

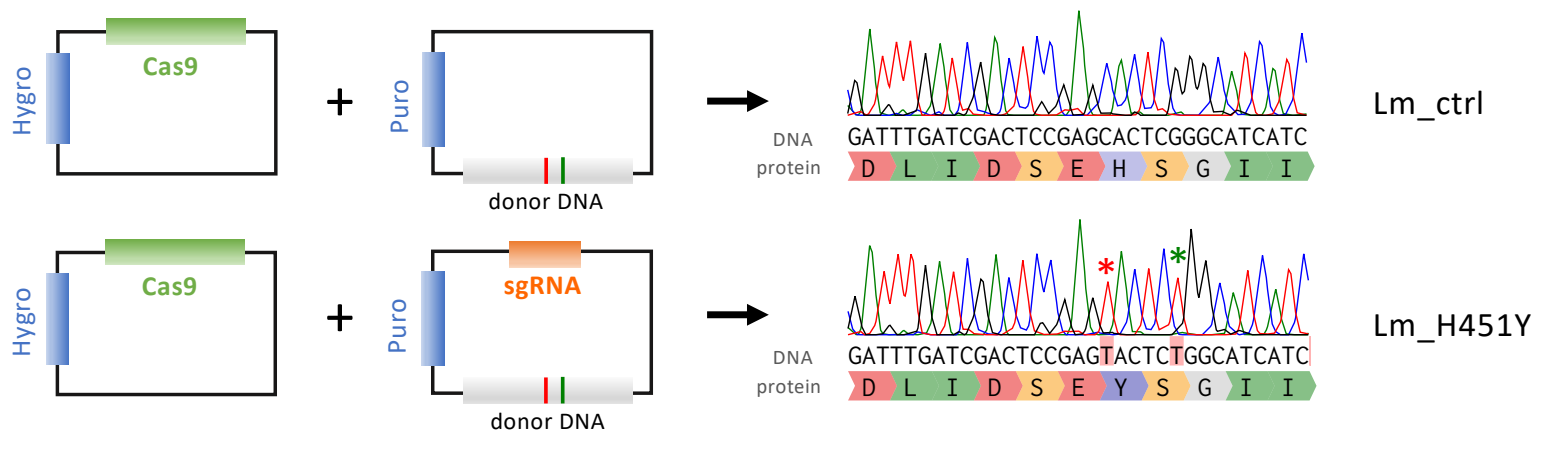

Figure 2 
(a)

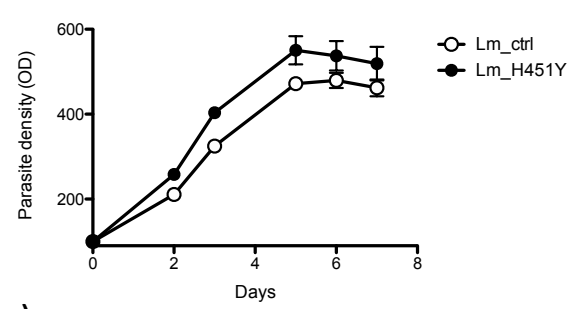

(c)

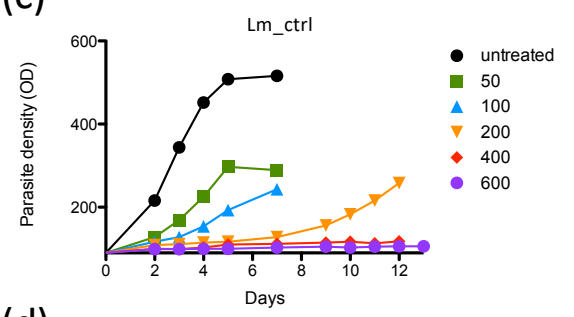

(d)

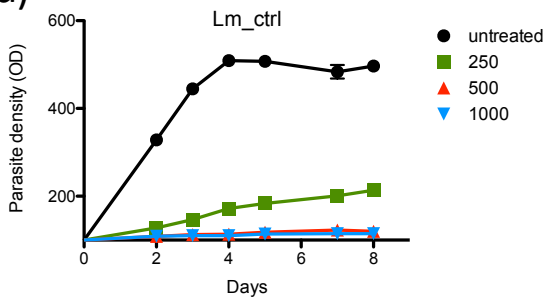

(e)

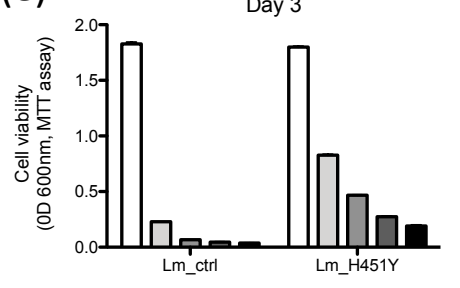

(h)

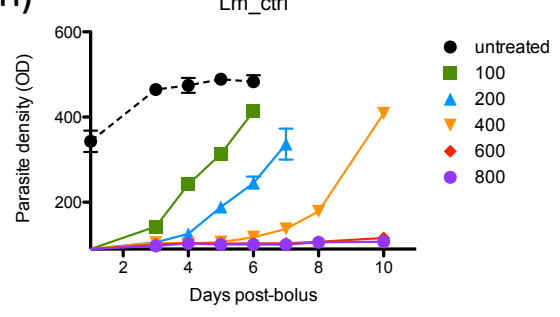

(b)
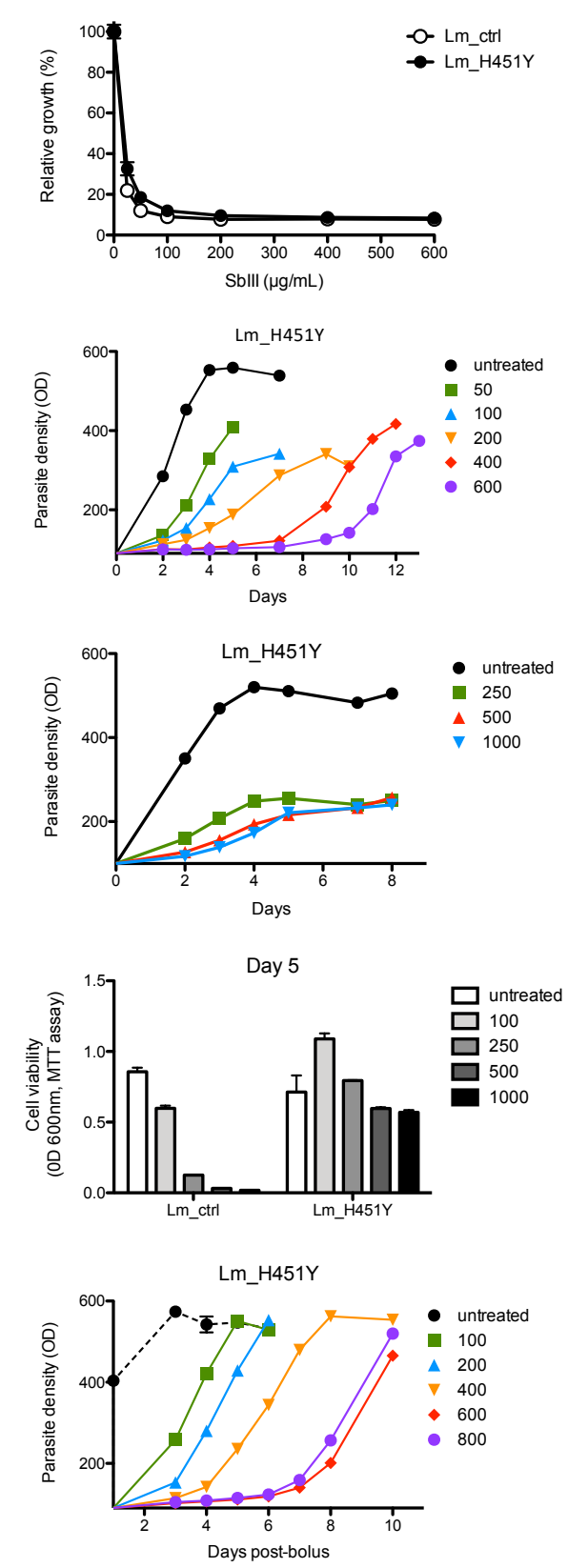

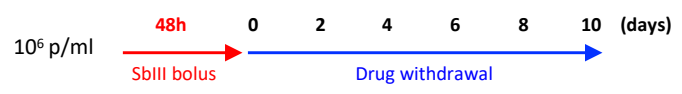

(f)
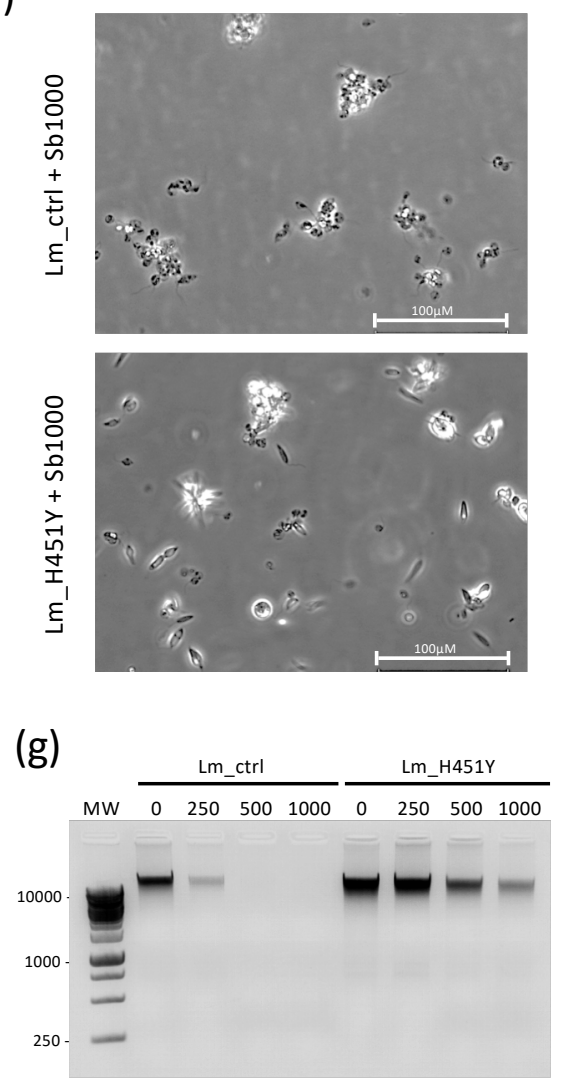

(i)

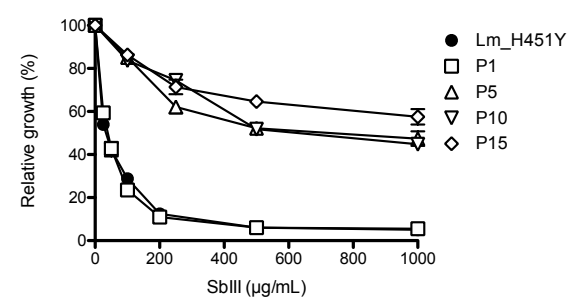

Figure 3 


\section{Supplementary data}

A single amino acid substitution (H451Y) in Leishmania calcium-dependent kinase SCAMK confers high tolerance and resistance to antimony. B Vergnes, E Gazanion, C Mariac, M Du Manoir, L Sollelis, JJ Lopez-Rubio, Y Sterkers, AL Bañuls.

Table S1. Primers used in this study.

\begin{tabular}{|c|c|}
\hline Primer name & Sequence (5'-3') \\
\hline \multicolumn{2}{|l|}{ Crispr/Cas9 } \\
\hline Lm_1F & CTGGTACCCATCACCCCTCAGATGAGC (Kpnl) \\
\hline Lm_1R & GATGATGCCAGAGTACTCGG \\
\hline Lm_2F & CCGAGTACTCTGGCATCATC \\
\hline Lm_2R & GCTCTAGAGTCGAGAAACCACTTGTGG (Xbal) \\
\hline seed_F & CAGGCACCGCTGGTGGtttgatcgactccgagcactGTTTTAGAGCTAGAAAT \\
\hline seed_R & ATTTCTAGCTCTAAAACagtgctcggagtcgatcaaaCCACCAGCGGTGCCTG \\
\hline LmiSCAMK_F & CAACAGCCATCTCACCGTGC \\
\hline LmiSCAMK_R & GGACAACGGGGTTTCACGTG \\
\hline \multicolumn{2}{|l|}{ qRT-PCR } \\
\hline gapdh_F & GACACGTCGATCCAGGAGAT \\
\hline gapdh_R & AGCCCCACTCGTTGTCATAC \\
\hline GSH1_F & ACTCCCGTACAATGCAGGAC \\
\hline GSH1_R & GTAGCAGGGGTGAAGAGCTG \\
\hline ODC_F & CGTCGACCTCTTCTTCCTTG \\
\hline ODC_R & TCTCGATCGCACTCTTGTTG \\
\hline TR_F & TGAACAGCATCAACGAGAGC \\
\hline TR_R & TTGCTCGTGATGCAGAACTC \\
\hline G6PיDH_F & TGCAGATCACGTTCAAGGAG \\
\hline G6PDH_R & CGGCTCAATGCACTTCAGTA \\
\hline NADK_F & GATCAGGAAGACCAGGTGGA \\
\hline NADK_R & ACAACTTGTGCTGCAAATCG \\
\hline GspdS_F & AGTCCGACGCTCTCTGACAT \\
\hline GspdS_R & GGAGACGTAGTGGTGGGAGA \\
\hline TRYS_F & AGCCGATGTGGAAAGTCATC \\
\hline TRYS_R & CCCATAGTTGCCACCAGACT \\
\hline TXN1_F & TTGAAGCTGCAGAAGCAGAA \\
\hline TXN1_R & GAAGTCСTCСTCСTCСTCGT \\
\hline MRPA_F & GGACGTGGAAGAGAGAGTCG \\
\hline MRPA_R & GTACTCGCCCATCAGAGAGC \\
\hline AQP1-F & GAACTTCACGTCGCAGAACA \\
\hline AQP1-R & GATGGCCATGTAGCTGGAGT \\
\hline
\end{tabular}


Table S2. Off-target mutation analysis. List of the off-target candidates identified with the Protospacer Workbench software (with RazerS 3, and five mismatches allowed).

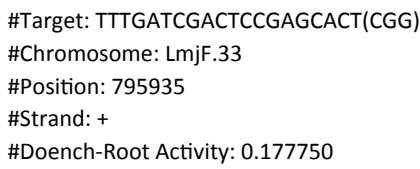

target

NGG PAM

non-canonical PAM

\begin{tabular}{|c|c|c|c|c|c|c|c|c|}
\hline chromosome & position & strand & sequence & pam & mismatches & dra & score & $\begin{array}{l}<20 \text { bp from a } \\
\text { specific INDEL in } \\
\text { Lm_H451Y }\end{array}$ \\
\hline LmjF.33 & 795935 & + & TTTGATCGACTCCGAGCACT & CGG & 0 & 0.17774975685470332 & 1.0 & \\
\hline LmjF. 25 & 827829 & - & CTTGATTGACGCAGAGCACT & TGG & 4 & 0.048381370162212375 & 0.0022051146716772154 & NO \\
\hline LmjF.31 & 1158568 & - & TTGGATCGACTCAGGGCAGT & AGG & 4 & 0.05825528097973003 & 0.0005192731021153846 & NO \\
\hline LmjF.30 & 1324740 & - & TGTGAACGACTCCGGGCTCT & GAG & 4 & 0.0 & 0.000512281696832579 & NO \\
\hline LmjF.36 & 706422 & + & CTTGAGCGACTCAGAGTAGT & AGG & 5 & 0.3237375902104075 & 0.00028025959499999994 & NO \\
\hline LmjF.16 & 692860 & - & TTTCATCGGCTCCGCGCCCA & AAG & 5 & 0.0 & 0.00012875219199189871 & NO \\
\hline LmjF.04 & 358636 & + & TTTCAGCGACGCTGAGCACG & AAG & 5 & 0.0 & 0.0005212929826708861 & NO \\
\hline LmjF.19 & 612710 & + & TTTCTTCGAATCCGACAACT & TAG & 5 & 0.0 & 0.000565260380487805 & NO \\
\hline LmjF.36 & 1305474 & - & CTTCATCGACTACATGCACT & GGG & 5 & 0.1253521096282401 & 0.00018433794370370374 & NO \\
\hline LmjF.35 & 576833 & + & TTTGATCGCCTGGGAGCAGG & AGG & 5 & 0.17388963529208032 & 0.00013826052225180001 & NO \\
\hline LmjF.27 & 942224 & + & ATTGAGCGCCGCCGTGCACT & CGG & 5 & 0.15677322326935583 & 0.0005158850416296295 & NO \\
\hline LmjF.33 & 796031 & + & TTCGATGGACACGGAGCACA & AGG & 5 & 0.04419561195415686 & 0.0005877012458031232 & NO \\
\hline LmjF.34 & 950998 & + & GTTGCGCGACTCCGAGTGCT & CGG & 5 & 0.2699483343642831 & 0.00044482702564102556 & NO \\
\hline LmjF.18 & 722184 & - & TTTATTCGACTCAGAGCGCA & CGG & 5 & 0.6556028597619067 & 0.0003042913397468355 & NO \\
\hline LmjF.30 & 370497 & - & GCAGAACGACTGCGAGCACT & AGG & 5 & 0.21520147439812617 & 0.002655410685714286 & NO \\
\hline LmjF.36 & 2457360 & + & TTGCAACGACACCGAGCACG & CAG & 5 & 0.0 & 0.0013451797386923078 & NO \\
\hline LmjF.36 & 1570009 & - & TTTGGTCTGCTCCGAGCCCG & GGG & 5 & 0.34862116391658615 & 0.0004744133939999999 & NO \\
\hline LmjF.18 & 575242 & + & TTTCATCCACGCCGTGCACG & TGG & 5 & 0.32245220558738613 & 0.0005966921620253165 & NO \\
\hline LmjF.27 & 636627 & - & TTTGTTCGACTCAGGGCGAT & CAG & 5 & 0.0 & $6.0081526399999974 \mathrm{e}-05$ & NO \\
\hline LmjF. 25 & 411227 & - & TATGATCGACTCCGTTCATG & TAG & 5 & 0.0 & $5.976304494545457 e-05$ & NO \\
\hline LmjF.08 & 529243 & + & TTTGATCCTCTCCGAGAGCA & GAG & 5 & 0.0 & 0.00017604737994216869 & NO \\
\hline
\end{tabular}

Table S3. Heterozygous and homozygous non-synonymous SNPs identified in the coding sequences of KO-SbR mutants but not in the $\Delta p n c 1$ parental line.

\begin{tabular}{|c|c|c|c|c|c|c|c|c|c|c|c|}
\hline \#CHROM & POS & GENE ID & PRODUCT DESCRIPTION & REF & ALT & AA change & QUAL & GT & DP & RO & AO \\
\hline LinJ.33 & 689935 & LinJ.33.1810 & protein kinase & C & $\mathrm{T}$ & H451Y & 376,64 & $1 / 1$ & 12 & 0 & 12 \\
\hline LinJ.29 & 279621 & LinJ.29.0790 & heat shock protein 90 (LPG3) & G & A & R592C & 293 & $0 / 1$ & 18 & 6 & 12 \\
\hline LinJ.15 & 428514 & LinJ.15.1070 & glutamate dehydrogenase & G & $\mathrm{C}$ & Q917E & 264,17 & $0 / 1$ & 24 & 12 & 12 \\
\hline
\end{tabular}

GT=Genotype, DP=Read Depth, RO=Reference allele observation count, AO=Alternate allele observation count 
Table S4. Non-synonymous SNPs identified in the coding sequences of Lm_H451Y-SbR mutants, but not in Lm_ctrl and Lm_H451Y parasites.

\begin{tabular}{|c|c|c|c|c|c|c|c|c|c|c|c|}
\hline \#CHROM & POS & GENE ID & PRODUCT DESCRIPTION & REF & ALT & AA change & QUAL & GT & DP & RO & AO \\
\hline LmjF.17 & 584469 & LmjF.17.1200 & hypothetical protein & C & $\mathrm{T}$ & A75V & 61,93 & $0 / 1$ & 10 & 6 & 4 \\
\hline LmjF.05 & 19975 & LmjF.05.0060 & major vault protein & $\mathrm{T}$ & A & S647T & 61,93 & $0 / 1$ & 10 & 6 & 4 \\
\hline LmjF.20 & 352500 & LmjF.20.0850 & pseudouridine synthase TruD & A & G & E288G & 60,29 & $0 / 1$ & 10 & 5 & 5 \\
\hline LmjF.34 & 1103815 & LmjF.34.2480 & hypothetical protein & G & A & stop & 55,44 & $0 / 1$ & 4 & 1 & 3 \\
\hline LmjF.30 & 915322 & LmjF.30.2380 & hypothetical protein & A & G & V255A & 53,61 & $0 / 1$ & 11 & 7 & 4 \\
\hline
\end{tabular}

GT=Genotype, DP=Read Depth, RO=Reference allele observation count, $A O=A$ Iternate allele observation count

Table S5. Results of the CNV-seq method used to compare CNVs in Sblll-sensitive (KO) and Sblll-resistant (KO-SbR) $\Delta p n c 1$ parasites.

\begin{tabular}{|c|c|c|c|c|c|c|c|}
\hline CNV & \#CHROM & START & END & SIZE (bp) & $\log 2$ & p.value & COMMENT \\
\hline CNVR_1 & 16 & 751 & 9750 & 9000 & -1.362885 & $1.155912 \mathrm{e}-231$ & nc tel \\
\hline CNVR_2 & 04 & 116251 & 122250 & 6000 & 0.7291408 & $8.793945 e-86$ & nc tel \\
\hline CNVR_3 & 04 & 467251 & 476250 & 9000 & -1.065832 & $8.215468 \mathrm{e}-111$ & nc tel \\
\hline CNVR_4 & 11 & 751 & 6750 & 6000 & -1.67662 & $1.353293 \mathrm{e}-199$ & nc tel \\
\hline CNVR_5 & 17 & 662251 & 668250 & 6000 & -1.155018 & $9.593298 \mathrm{e}-88$ & nc tel \\
\hline CNVR_6 & 24 & 863251 & 869250 & 6000 & -0.8339029 & $7.110614 \mathrm{e}-53$ & nc tel \\
\hline CNVR_7 & 32 & 751 & 6750 & 6000 & -1.397065 & $8.888547 \mathrm{e}-158$ & nc tel \\
\hline CNVR_8 & 35 & 219751 & 225750 & 6000 & -0.8113096 & $4.357229 \mathrm{e}-54$ & part of LinJ.35.0530 (ppg5) \\
\hline CNVR_9 & 18 & 716251 & 722250 & 6000 & -1.402387 & $1.036969 \mathrm{e}-108$ & nc tel \\
\hline CNVR_10 & 22 & 653251 & 660750 & 7500 & -1.008428 & $2.584667 e-92$ & nc tel \\
\hline CNVR_11 & 02 & 326251 & 335250 & 9000 & -0.7970366 & $1.0524 \mathrm{e}-81$ & nc tel \\
\hline CNVR_12 & 12 & 751 & 8250 & 7500 & -1.738381 & 0 & nc tel \\
\hline CNVR_13 & 23 & 751 & 6750 & 6000 & -1.995511 & 0 & nc tel \\
\hline CNVR_14 & 30 & 1358251 & 1367250 & 9000 & -1.276207 & $4.045936 \mathrm{e}-143$ & nc tel \\
\hline CNVR_15 & 26 & 1046251 & 1052250 & 6000 & -0.8611537 & $1.727647 \mathrm{e}-56$ & nc tel \\
\hline CNVR 16 & 13 & 543751 & 549750 & 6000 & -1.736291 & $4.360188 \mathrm{e}-204$ & nc inter \\
\hline
\end{tabular}

nc tel: non-coding telomeric extremity ; nc inter: non-coding intergenic sequence ; ppg5: member of the large and conserved family of proteophosphoglycan. 


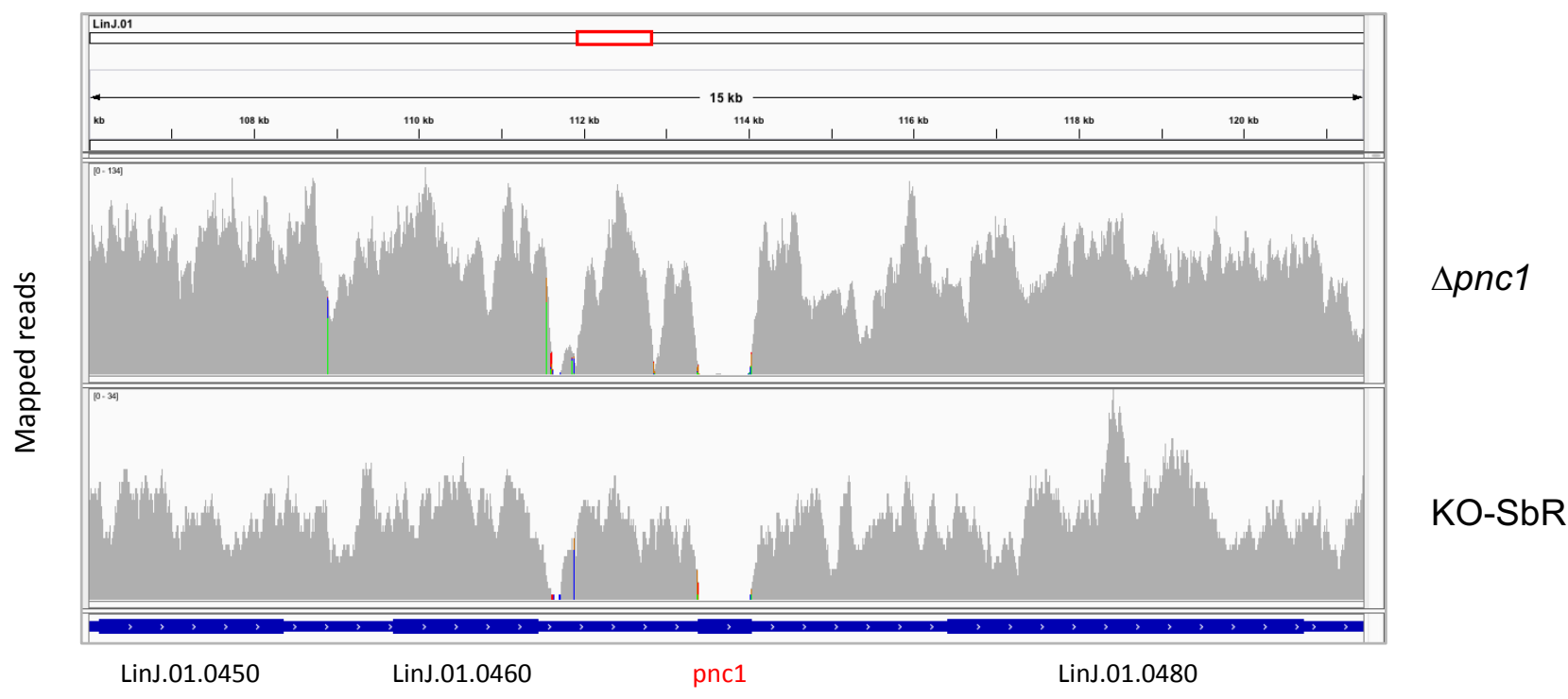

Figure S1. Validation of pnc1 gene inactivation in sequenced strains. Read coverage (bam files) along chromosome 1 in the $\Delta p n c 1$ and KO-SbR strains showing the lack of aligned reads for the LinJ.01.0470 gene (pnc1). 

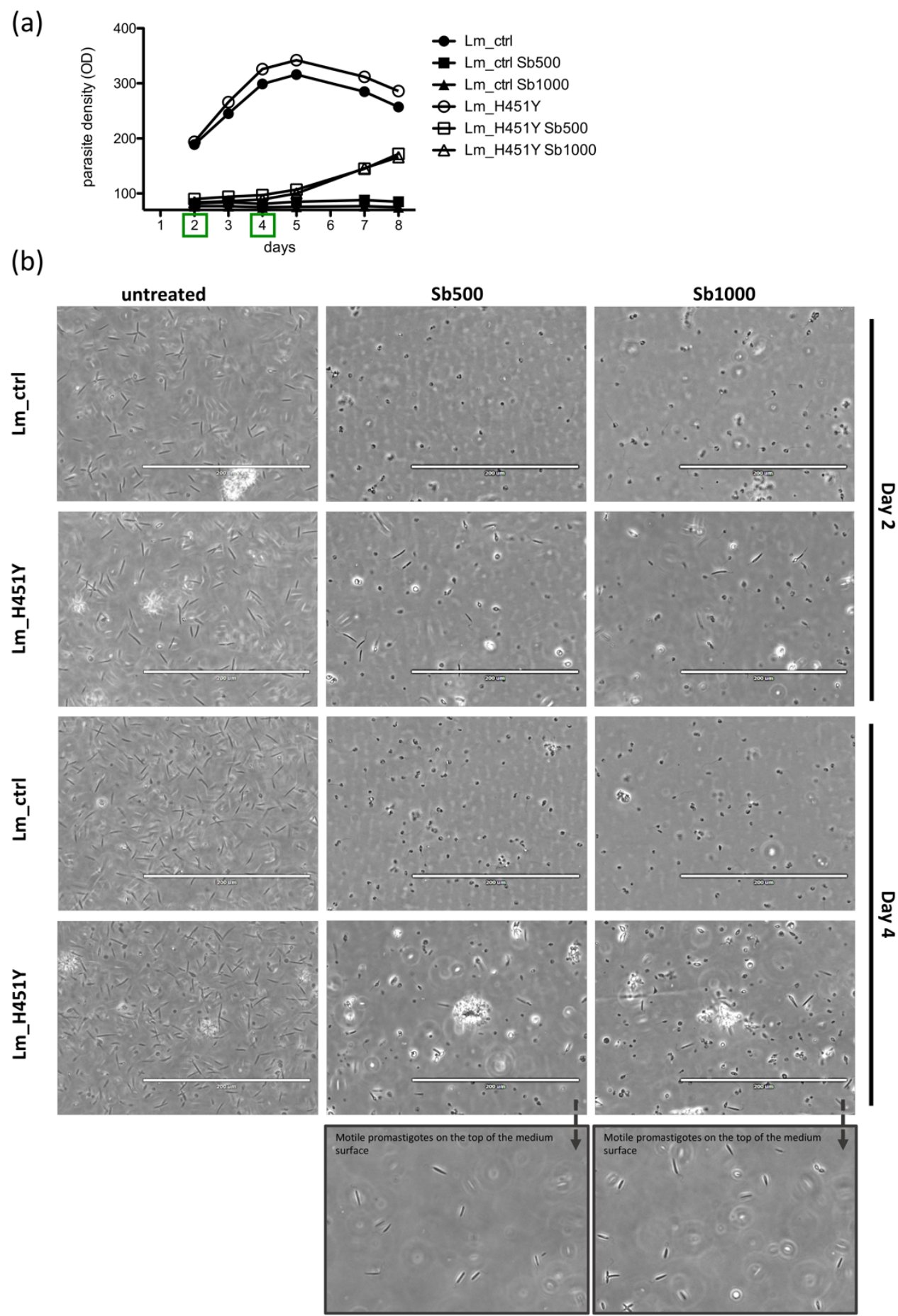

Figure S2. Action of cytocidal concentrations of Sbll on control and edited parasite populations. (a) Follow-up of parasite density in control (Lm_ctrl) and edited (Lm_H451Y) populations seeded at $4.10^{6}$ parasites $/ \mathrm{mL}$ and exposed to cytocidal concentrations of SbIII (500 and $1000 \mu \mathrm{g} / \mathrm{mL}$ ). (b) Images of the parasite cultures described in (a) taken after 2 and 4 days of growth (EVOS FL inverted microscope with x20 magnification). 

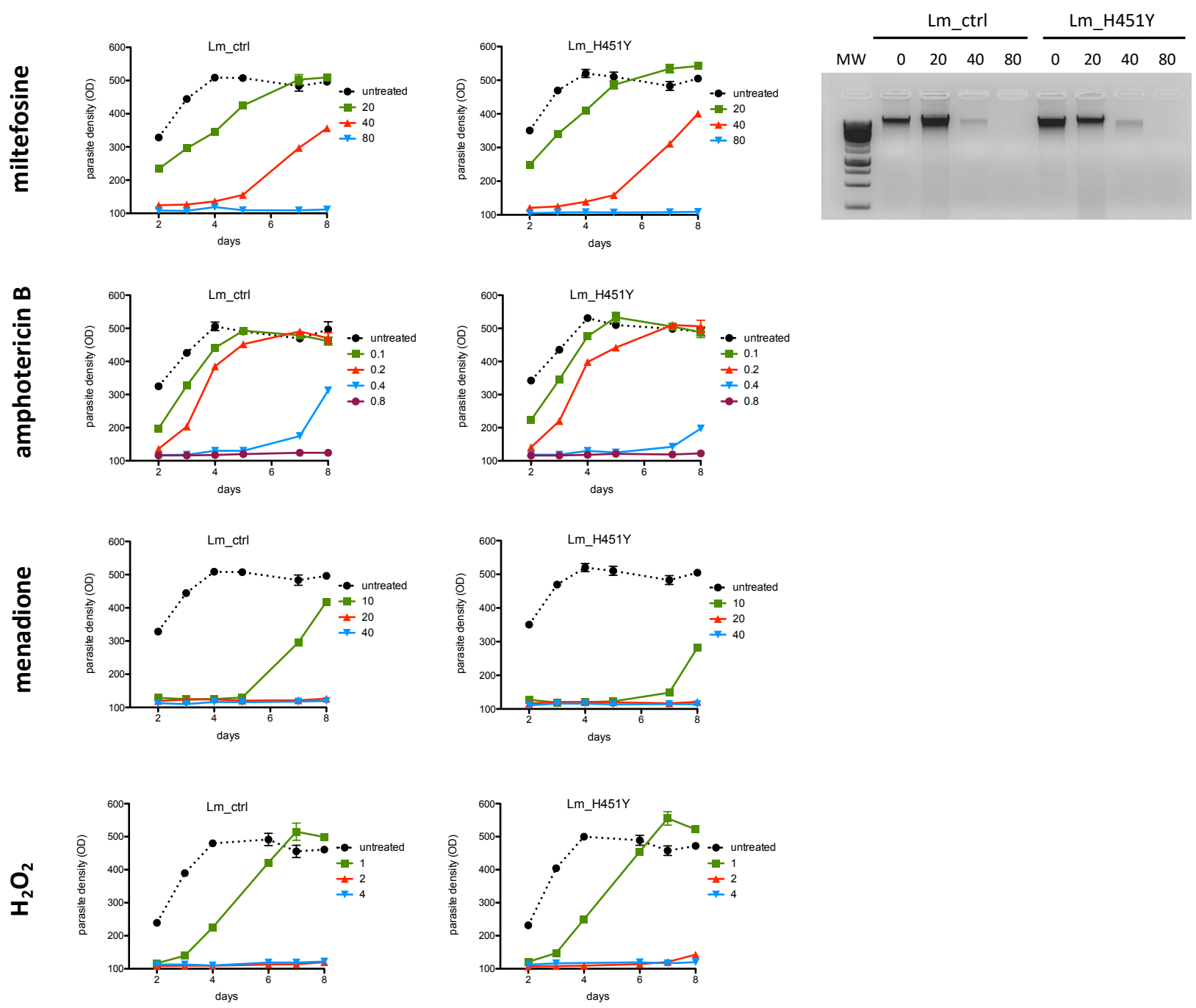

Fig. S3. Growth kinetics of $\mathrm{Lm}$ _ctrl and $\mathrm{Lm}$ _H451Y parasites exposed to different drugs that induce ROS formation. Similarly to the experiments presented in Fig. 3d, parasites were seeded at $4.10^{6}$ parasites $/ \mathrm{mL}$ and incubated with cytocidal concentrations of miltefosine $(20,40$, $80 \mu \mathrm{M})$, amphotericin $\mathrm{B}(0.1,0.2,0.4,0.8 \mu \mathrm{M})$ or menadione $(10,20,40 \mu \mathrm{M})$. Parasite density was determined daily by $\mathrm{OD}$ measurement. For $\mathrm{H}_{2} \mathrm{O}_{2}$, parasites were incubated with 1,2 , or $4 \mathrm{mM}$ $\mathrm{H}_{2} \mathrm{O}_{2}$ for $1 \mathrm{~h}$, then washed and resuspended in fresh medium. The integrity of genomic DNA extracted from Lm_ctrl and Lm_H451Y cultures exposed to 20, 40 or $80 \mu \mathrm{M}$ miltefosine for three days was analysed as described in Fig. $3 \mathrm{~g}$. 